\title{
Landau Levels in Two and Three-Dimensional Electron Gases in a Wide Parabolic Quantum Well
}

\author{
C.S. Sergio, G.M. Gusev, J.R. Leite, \\ Instituto de Física da Universidade de São Paulo, SP, Brazil \\ E.B. Olshanetskii, A.A. Bykov, N.T. Moshegov, A.K. Bakarov, A.I. Toropov, \\ Institute of Semiconductor Physics, Novosibirsk, Russia \\ D.K. Maude, O. Estibals, and J.C. Portal \\ GHMF, MPI-FKF/CNRS, BP-166, F-38042, Grenoble, Cedex 9, France
}

Received on 23 April, 2001

\begin{abstract}
Shubnikov-de Haas oscillations are measured in a wide parabolic quantum well with 6 subbands in a tilted magnetic field. We find two types of oscillations. The oscillations at low magnetic field are shifted towards higher fields with tilted angles, and can be attributed to the two-dimensional Landau state at the bottom subband. The position of the second type oscillations do not shift with tilted angles indicating a three-dimensional character of the Landau state formed by the highest subbands. The bottom level in the quantum well is not overlapped with the highest subbands due to the enhanced quantum scattering time of the lowest subbands.
\end{abstract}

\section{Introduction}

When a magnetic field is applied in a bulk semiconductor, the free eletrons which carry the eletric charge perform an orbital motion in the plane perpendicular to the magnetic field direction. This motion becomes quantized, and equally spaced levels (the Landau levels) separated in energy by $\hbar \omega_{c}$ are formed. The energy of the system is given by

$$
E_{i}=\left(i-\frac{1}{2}\right) \cdot \hbar \omega_{c}+\frac{\hbar^{2} k_{z}^{2}}{2 m}
$$

where $i=1,2,3, \ldots$ is the Landau quantum number, $m$ is the effective mass of the electron, and $\omega_{c}=e B / m$ is the cyclotron frequency.

The electrons within one Landau leavel may be considered to behave as if they were one-dimensional. The density of states (DOS), $\varrho(E)$, which in the abstance of a magnetic fild is a parabola given by $\varrho(E) \propto E^{1 / 2} d E$, now becomes the sum of a set of one-dimensional densities of states, where $\varrho(E) \propto E^{-1 / 2} d E$, each starting at the bottom of a Landau level. The very sharp singularities at the bottom of each Landau level is the origin of the Shubnikov-de Haas ( $\mathrm{SdH}$ ) effect. In practice these sharp features are smeared out by scattering.

When free particles are confined to a small region of space, either by a potential barrier formed by physical boundaries of the sample, the energy levels of the particles become quantized due to the wave-like behavior of the particles. Are of the simplest example of this is a square well potential. For a square well of width $w_{e}$ the energy of the bound states are given by (infinite potential barrier)

$$
E_{n}=\frac{n^{2}\left(h / w_{e}\right)^{2}}{8 m}
$$

where $n=1,2,3, \ldots$ is the subband index. We see that the energy separation increases from the bottom to top levels with subband number.

If a magnetic field is applied perpendicular to the two-dimensional (2D) electron gas, then a total quantization of the electron levels takes place. The resulting DOS consists of a set of $\delta$-functions separated by $\hbar \omega_{c}$, in the absence of scattering. When scattering is present each $\delta$-function broadens into peaks with width $\Gamma$.

In present the work we study remotely doped $4000 \AA$ parabolic quantum well (PQW) with intermediary density, which allow us to obtain 6 occupied subbands (for full case we have 8 subbands occupied). In order to characterize the wide parabolic well and determine the subband structure we measure $\mathrm{SdH}$ oscillations in a tilted magnetic field. The oscillations contain two frequencies, one depends on the tilt angle, and other does not. We attribute such behaviour to the threedimensional (3D) Landau states formed by the 5 higher subband and 2D Landau states originated from the lowest subband.

\section{Experiment and Discussion}

The samples used are the GaAs/ $\mathrm{Al}_{x} \mathrm{Ga}_{1-x} \mathrm{As}$ PQW grown on undoped (100) GaAs substrate by molecularbeam epitaxy. On the top of the substrate there is 
$10,000 \AA \mathrm{GaAs}$ buffer layer with 20 periods of $\operatorname{AlAs}(5$ $\mathrm{ML}) \mathrm{GaAs}(10 \mathrm{ML})$ superlattice, followed by $5000 \AA$ $\mathrm{Al}_{x} \mathrm{Ga}_{1-x} \mathrm{As}$ with $x$ varying from 0.07 to 0.27 . The structure consists of a 4000 - $\AA$-wide $\mathrm{Al}_{x} \mathrm{Ga}_{1-x}$ As well in which $x$ was quadratically varied between $x=0$, at the center of the well, and $x=0.19$, at the edges of the well. On each side, the well is bounded by Sidoped $\left(\sim 5.0 \times 10^{11} \mathrm{~cm}^{-2}\right) \mathrm{Al}_{0.3} \mathrm{Ga}_{0.7}$ As layers, grown next to spacer layers. The thicknesses of the undoped $\mathrm{Al}_{0.3} \mathrm{Ga}_{0.7}$ As space layers are $100 \AA$. A $100 \AA$ GaAs cap layer was grown as final layer of the estructure.

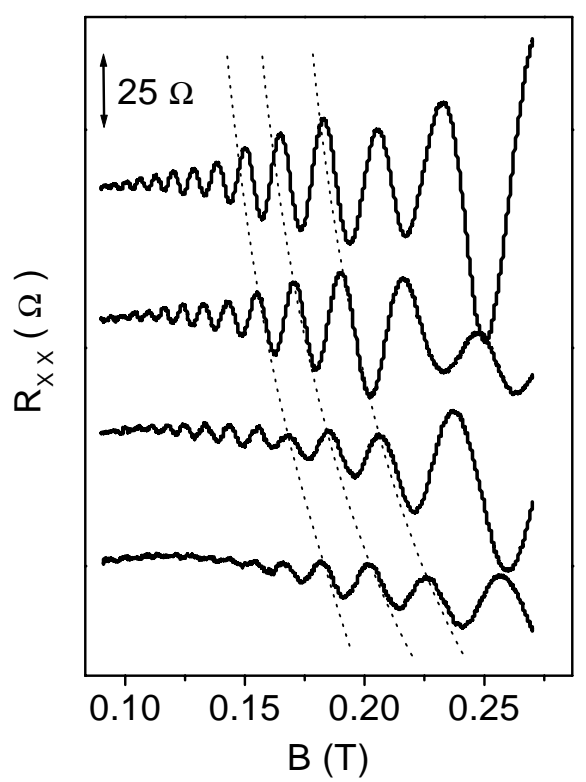

Figure 1. Low field part of the magnetoresistance oscillations as a function of the magnetic field, for different angles $\theta$ - top $(\theta=0)$, botton $(\theta=50)-, T=50 \mathrm{mK}$.

After growth, are photolithographically defined Hall bar with dimensions 100 X $200 \mu \mathrm{m}$. Four-terminal resistance and Hall measurements were made down to $50 \mathrm{mK}$ in magnetic field up to $17 \mathrm{~T}$. The measurements were performed with an ac current not exceeding $10^{-8} A$. Resistance was measured for different angles $\theta$ between the field and substrate plane in magnetic field using an in situ rotation of the sample.

The mobility of the electron gas in the well is $\mu_{H}=210 \times 10^{3} \mathrm{~cm}^{2} / \mathrm{Vs}$, and the electron concentration is $n_{H}=2.5 \times 10^{11} \mathrm{~cm}^{-2}-$ from the Hall effect at low field.

Three dimensional pseudocharge is $N_{+}=0.9 \times$ $10^{16} \mathrm{~cm}^{-3}$ which corresponds to the classical width of the $3 \mathrm{D}$ electron gas $w_{e}=n_{H} / N_{+}=2900 \AA$. We perform the numerical self-consistent calculations for PQW of width $W=4000 \AA$, which yields the following energies for the first 6 electric subbands (in $m e V): E_{1}=0.05 ; E_{2}=0.21 ; E_{3}=0.46 ; E_{4}=0.80$; $E_{5}=1.22 ; E_{6}=1.73 ;$ and $E_{F}=2.03 \mathrm{meV}$ (for $\left.m=0.075 m_{0}\right)$.
Fig. 1 shows the low field dependence of the $\mathrm{SdH}$ oscillations for different angles $\theta$. The oscillations are periodic in $1 / B$ and contain only single frequency. The position of the oscillations are shifted, as expected or $2 \mathrm{D}$ electron gas, when magnetic field is tilted from the normal to the substrate. The magnetoresistance are very well described by the conventional formula for the $\mathrm{SdH}$ oscillations in the 2D case: [1]

$$
\begin{aligned}
\frac{R_{x x}-R_{0}}{R_{0}} & =4 \cdot \frac{A_{T}}{\sinh A_{T}} \cdot \exp \left(-\frac{\pi}{\omega_{c} \cdot \tau}\right) \\
& \times \cos \left(\frac{2 \pi E_{F(2 D)}}{\hbar \omega_{c}}-\pi\right)
\end{aligned}
$$

where $A_{T}=\left(2 \pi^{2} k_{B} T\right) /\left(\hbar \omega_{c}\right), \tau$ is a quantum lifetime, $E_{F(2 D)}$ is the Fermi energy of the 2D level, and $R_{0}$ represents the classical resistance in zero applied field.

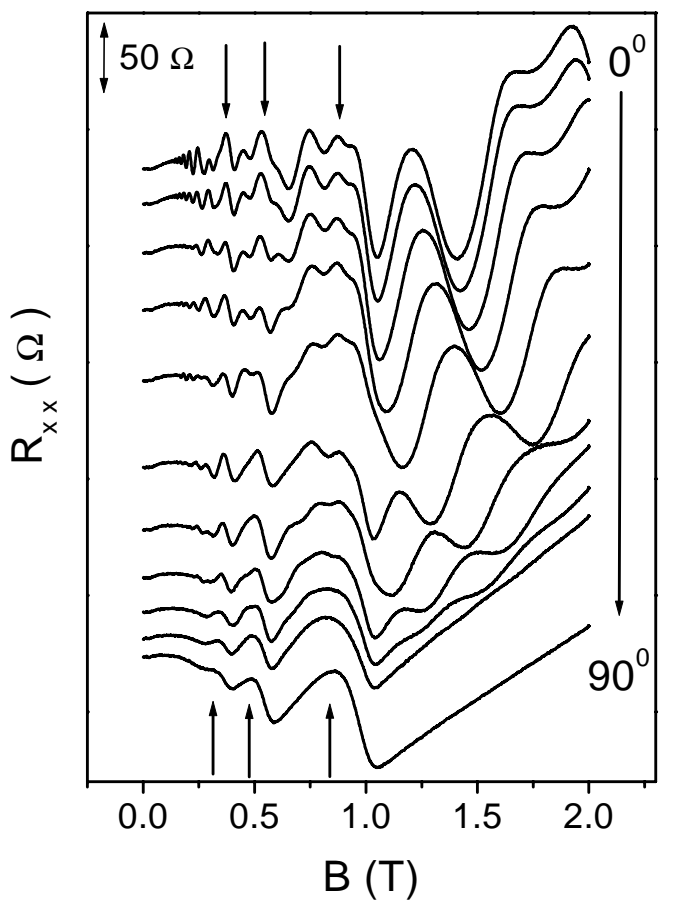

Figure 2. The magnetoresistance oscillations as a function of the magnetic field up to $3 T$ for different angles $\theta$, $T=50 \mathrm{mK}$. Arrows: position of the 3D Landau states.

From the comparison of the experimental SdH oscillations (Fig. 1, $\theta=0$ ) and Eq. 3 we extract the carrier density $n_{s 1}=0.7 \times 10^{11} \mathrm{~cm}^{-2}$, which is coincident with $2 \mathrm{D}$ electron density obtained from the calculation for the lowest subband. Surprisingly, we don't see any contribution at this magnetic field from the second subband.

Fig. 2 shows $R_{x x}(B)$ extended to the magnetic field up to $3 T$. We can see 3 oscillations indicated by arrows. Surprisingly, the position of these oscillations does not depend on the tilt angle. We attribute such behaviour to the formation of the $3 \mathrm{D}$ Landau states. 
In real systems the energy levels will have finite widths because of the disorder, therefore corresponding electric subbands can overlap. Naively, it is expected that the lowest subbands will overlap first, when the width of the well increases, because the distance between levels $\Delta_{i j}=E_{j}-E_{i}$ grows up as the square of the index number. However, if the broadening of the levels $\Gamma_{j}$ increases faster than $\delta_{i j}=\Delta_{i j} / 2$ the highest electric subbands are collapsed to the bulk Landau states before the lowest one. Therefore the specific features of the investigated wide PQW is a coexistence $3 \mathrm{D}$ and $2 \mathrm{D}$ electron states inside of the well. In the tilted field $2 \mathrm{D}$ $\mathrm{SdH}$ oscillations are shifted to the higher magnetic field, and can cross 3D SdH peaks, which does not depend on the tilt angle.

The theoretical expression for the SdH oscillations in $3 \mathrm{D}$ case is slightly different from the $2 \mathrm{D}$ case: [2]

$$
\begin{aligned}
\frac{\Delta R_{x x}}{R_{0}} & =\frac{2}{5} \cdot\left(\frac{\hbar \omega_{c}}{2 E_{F(3 D)}}\right)^{1 / 2} \cdot \frac{A_{T}}{\sinh A_{T}} \\
& \times \exp \left(-\frac{\pi}{\omega_{c} \cdot \tau}\right) \cdot \cos \left(\frac{2 \pi E_{F(3 D)}}{\hbar \omega_{c}}-\frac{\pi}{4}\right)
\end{aligned}
$$

Fits the experimental curve for $3 \mathrm{D} \mathrm{SdH}$ oscillations to the Eq. 4 give the value $E_{F(3 D)}=1.88 \mathrm{meV}$. From this value we find the bulk concentration for highest subbands $N_{(3 D)}=0.7 \times 10^{16} \mathrm{~cm}^{-3}$.

The density profile for the 5 higher subbands is not a constant and has a deep minimum in the center as we can see in Fig. 3. Therefore the sheet density can not be recalculated from the equation $n_{s}=w_{e} \cdot N_{(3 D)}$. The width of the self-consistent electron density profiles can be defined as:

$$
\left(w_{e f f}\right)^{2}=\frac{12}{n_{s}} \int_{0}^{W}\left(z-\frac{W}{2}\right)^{2} n(z) d z
$$

where $n(z)=\sum n_{s i}\left|\phi_{i}(z)\right|^{2}$, and $\phi_{i}$ is the envelope function of the electrons in the $i$ th subband.

The sheet density of the electrons in the 5 highest subband is $\left(n_{H}-n_{s 1}\right)=1.8 \times 10^{11} \mathrm{~cm}^{-2}$. We obtain the self-consistent value $w_{e f f}=2600 \AA$ and find bulk density for the quasi-three-dimensional subband $N_{(3 D)}=\left(n_{H}-n_{s 1}\right) / w_{e f f}=0.7 \times 10^{16} \mathrm{~cm}^{-3}$, which is equal than the bulk density determined from the measurements of the 3D SdH oscillations.

Furthermore, we calculate $\tau$ following the formalism of the Ando and Gold taking into account the influence of the intersubband coupling on the screening and correlation corretions. [3] We consider only two major scattering mechanisms - remote and background impurity scattering. The results of the level broadening $\Gamma_{i}=\hbar / 2 \tau_{i}$ are, in meV: $\Gamma_{1}=0.06 ; \Gamma_{2}=0.08$;
$\Gamma_{3}=0.18 ; \Gamma_{4}=0.21 ; \Gamma_{5}=0.25 ;$ and $\Gamma_{6}=0.40$. Our empirical finding is that $\Gamma_{2}<\delta_{12}$ for $2 \mathrm{D}$ confinement effects to be observable in botton subband. We obtain $\Gamma_{2} \approx \delta_{12}$. For highst subbands $\Gamma_{j}>\delta_{i j}$ - therefore theses subbands are overlapped and form the $3 \mathrm{D}$ system.

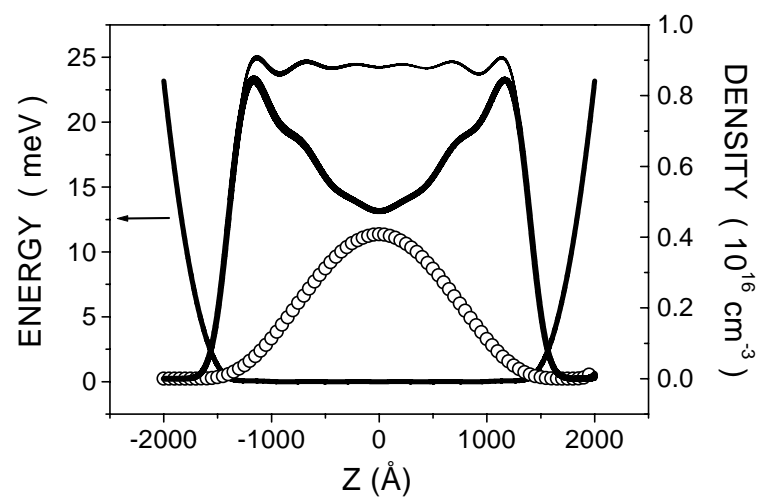

Figure 3. Electron density profile as a function of position in the well for 5 top subbands (solid line) and bottom subband (circle). The thick line: total density profile.

\section{Conclusions}

In the present work we realize the system with $2 \mathrm{D}$ and quasi-3D electron gas coexisting in the same quantum well. We use standard analysis of SdH oscillations in the tilted magnetic field and explore the fact that $2 \mathrm{D}$ Landau states are sensitive to the perpendicular magnetic field. We evaluate the broadening of the levels due to remote and background impurity scattering in the presence of the intersubband scattering and find that the bottom subband is not overlapped with the highest subbands. Therefore, the $2 \mathrm{D}$ state belongs to the lowest subband and the 3D state, to the highest subband.

It is known that $2 \mathrm{D}$ and $3 \mathrm{D}$ systems obey several properties, which are radically different, such as localization in random potential. We believe that our system can be used for comparing such effects.

\section{Acknowledgments}

We would like to thank FAPESP for financial support.

\section{References}

[1] T.Ando, J.Phys.Soc.Jpn , 37, 1233 (1974).

[2] Landau Level Spectroscopy, Modern Problems in Condensed Matter Sciences, edited by G. Landwehr and E.I. Rashba, NORTH-HOLLAND, Volume 27.2 (1991).

[3] A Gold, Phys. Rev. B, 35, 723 (1987); P.T.Coleridge , Phys. Rev. B, 44, 3793 (1991); E.Zaremba, Phys. Rev. B, 45, 14143 (1992). 